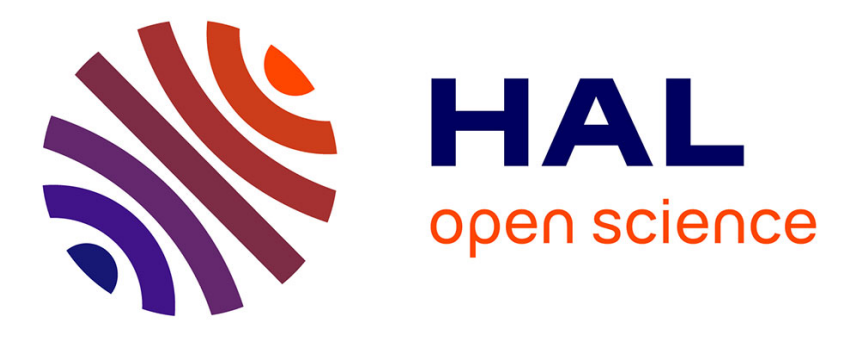

\title{
Évaluation locale d'un problème global: la représentation sociale du changement climatique en France et au Groenland
}

\author{
Elisabeth Guillou, Isabelle Richard, Weiss Karine
}

\section{- To cite this version:}

Elisabeth Guillou, Isabelle Richard, Weiss Karine. Évaluation locale d'un problème global: la représentation sociale du changement climatique en France et au Groenland. Bulletin de psychologie, 2017, Le rôle de l'incertitude dans la construction sociale des problématiques environnementales, 70-2 (548), pp.117-129. 10.3917/bupsy.548.0117 . hal-01676896

\section{HAL Id: hal-01676896 https://hal.science/hal-01676896}

Submitted on 7 Feb 2018

HAL is a multi-disciplinary open access archive for the deposit and dissemination of scientific research documents, whether they are published or not. The documents may come from teaching and research institutions in France or abroad, or from public or private research centers.
L'archive ouverte pluridisciplinaire HAL, est destinée au dépôt et à la diffusion de documents scientifiques de niveau recherche, publiés ou non, émanant des établissements d'enseignement et de recherche français ou étrangers, des laboratoires publics ou privés. 


\title{
Évaluation locale d'un problème global : la représentation sociale du changement climatique en France et au Groenland
}

\author{
MiCHEL-GUILLOU Élisabeth * \\ RICHARD Isabelle ${ }^{\text {b }}$ \\ WeIss Karine ${ }^{b}$
}

“ Centre de recherchtes en psychatogiz, cognitien el comnitutication (CRPCC - EA 1285)/ Inslitut des sciences de l'homme er de la société / Université de Bretogne occidentale/ Universilce earopeenne de Bretagne, France.

"Liboratoire fie psychologie sociale, EA 849 , Aix-Marseille Universite /Université de Nirmes, France.

Correspondance : Erisobeth Michel-Guillou, CRPCC. 20 rue Duquesne, CS 93837, 29238 Brest Cedex 3, France.

Courriel : elisabth.ntchel-guillou@umiv-brest.ff

\begin{abstract}
Itćsumé : L'existence d'un changement du clinyl fajt consensus au seir de ta communate scientificue. Elte implique ì ja lois ane gostion politique intemationale ot une prisc de conscience à lous les niveaux sociaux et inslítiofioncls.

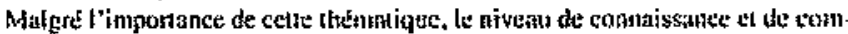

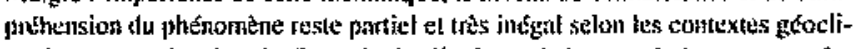

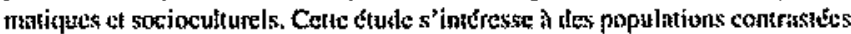
atin de recueillir teurs connaissances et rejpésentalions du chingenent clima-

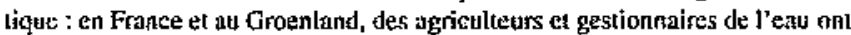
acé inkerrogds à l'aide d'cntretiens seni-directifs. Pour l'ensemble des enquélés, i] ressort que le changement climalique et ses conséquences sont perçus (réclbutffement, śéchturesses, elc.). A l'évidence, des diffísences apparaissent dass la pereeptien des morlificalions clinatiques tocales selon fes contex tes gćoclimatiques. Mais globulement, pour les inlerviewés, la gestion diu phénomène resle problénatigue er, notamnent, wa terures te projection dans l'awenir.
\end{abstract}

Locul Evaluation of a Glubal Problem: The Social Representation of Cthate Change in France and Greenland

Abstrat: Nowadays, there is consensus within the sciend ific community about the existence of climate change. [t entails both international policy manatsement and awareness at af social and institutionat levels. Despile the imaportance of this isster, the kevel of kacwledge and understanding of the phenomenon is still very partial and anequal, depending on the geoclimatic and socioculturat context. Therefort, we were iaterested in contrasting poputations in order to study their knowledge ant representations of climate change: farnters and water nanagers were qutustioned in France and Greentand using semistuctured interviews. The results show that, in both cotanites, clinunte change and its consequences are being aoticed (warming, drowghts, etc), Obwiously, differences appear in the percepliotn of Jocal wealther changes according to geoclinatic conlex r. Bur for everyon interviewed, climate-chonge managcatent remains problemalic, partictharty when is comes to ntaking projections for line future. 
Lexistence d'une modification du climal a'vest plus à prouver. Au sein de la communats scientifique la téalite d'une évolution rapide du chimat depuis la seconde moitie dis sibcle dernier et son origine en grande partie anthropique ront ntantunant conseusus (Brenchin, 2003 : JPCC. 2013 ; Liverman, 2007 : Oreskes, 2004). Mais, malgré cete entente daps le selts commun, de nomimeuses divergences persisien, notamment sur les const; guences du phenomène (modalites. echefle spaliotemporelle...). Airsi. forsqu'it s'agit. pour les personuss now scientifig̨ucment averties, $\mathrm{d}^{*}$ identifier lodalement les chatngements, la réaljte se róvèle complexe. Produit de l"activite humajne ou cycle maturel ? Recharfernest notoire ou simples variations du climas ? Marquage ou ahsence de stisons? Tout ust sujet a question. Le changement elimalique devient alors f"ohjet d'enjetx. de discussions. to de débals au sein desquels certains milltent pour des changements profonds de comportement ti de mote de vic, comple tertu le la gravite du phéno-

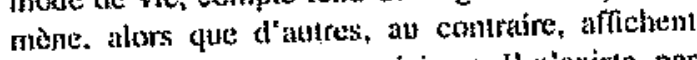
leur indécision ou leur scepticisme. Il a"existe. par conséquen, pas d'upirtion majoritaire etommune sur ce theme (Spence, Pidgeon, 2009), qui dovient source de conflis selon les grotpes et leturs piises de position.

Dans cel article, nous nous proposons d'analyser les enjeux lits au changement climatique en France el au Groenland. Les vartiahles culturethes, stiscep. tibles de prodluire des apprehensions spéciliques de cette question dans chaque pays, seront eludies. alin de saisir tes strategies developpeses pour faire face au probleme. Les auteurs de cel articles" interessent, ainsi, à fa manière don des individus clirestement en prise avec l'environement (agriculteurs st gestionmaires de l'cau) se representent te changement climatique : à quetle « rialites "ce phénomène renvoie-L-i] ? Quels sant les cojjeux pour chague population el dans chaque pays ?

\section{CONCEVOLR LE RAPPORT DE LHIOMME AU CHANGEMENT CLIMATIQUE: UNE PERSPECTIVE PSYCHO-SOCIO- ENVIRONNEMENTALE}

Définir le « changement climatique " r'est donc pas chose aisede. Le Groupement intergouvernemental sur l'Evolution du climat (GIEC) te definit contme o une variation de l'etal du climal que I'on peul décelet (...) par des modificalions de la moyenne edou de ta variabilite de ses proprietes et qui persiste pendant une longue periode, genéralenent pendant des decenthies nu plus. Il se rapporte à tout changernent du climat dans le tumps, gu' it soit do à la variabilité naturelle ou à l' activité Jumaine * (GIEC. 2007. p. 30). Bien que ta catsonlité humane ne soil qu'but variable envisagce dans la cḱfinilion. l'impace des activites humaines, par le biais de la production de gaz at offer de sorre, sir les modjicalions du climal, est ineonlestable scton les auteurs ke cet rapport : cette position esl chuirement affirmese au sein du derniter mponl (IPCC, 2013). En oucle, en prlus d'en être la cause, l'homme en subiı indéqiablement les constiquences. Dil aiflsi, l'existente dur lien entre fhomme et fo changement climatique est une évidence.

De oe lnit, cette retation el cel objel apparassent particulièrement pertinents pour la psychologite environnementale, gui porte sur te bien-ête el le comportemeat humain en relation taves sor enviroanement physigte et social, el qui prend en cons:dération la dimension temporelle et cuhturelle datts l'explicution des transactions entro l'hornme ta son environnement (Legendre, 2005 ; Moser. 2009 ; Stokols, Ajtmat1, 1987). La dimension spatiale est particulibrement importante dans l'évaluation des problenes enviromementaux. Les problentes planélaires sout, sous certains aspects. perçus comnc plus graves que les prothèmbs locaux, mais c'est àt cet nivealu glotral que les individus s'estimem individuellement les moins responsables (Garcial Mira, Real, Romay, 2005 ; Uzzelf, 2000). En oulre, I"Lvaluation de ces problànes est lide au degré do controble percu (Garcia-Mirn ef coll., 2005: Moser. 2009) : plus l'individu situe les problemes commo Eloignés de lui (de sa personns) ou de sof terriloire. et plus le contróle perçt sur les problemes environfenentaux appatraft hypothétique. La dimension temporelle jous egalement un rôle, puisque les individus undent à atre plus concemós par des problemes qu'ils siluent dans le présent que par des problènes dont l'impact semble plus inquicianc dans l'avenir (Gurcia+Mira el coll, 2005). Ces differentes dimensions, intervenant dans l'evaluat" ton des problematiques environnementales, ont cé modéliseses au travers de la thetorit des niveaux de construits (Libermath, Trope, 2008: Trope, Lifermal1, 2003 ; Trope, Libennan. Wakslak, 2007). Celke therie suggere que le niveat de represeptation (coneret ou abstruil) d'un objet ou d"un Evenement ast determine par ene distance psychologique, fonction de la distante spatiale (éloignte ou proche). (ampare]le (passće. fuiture, prosents), sociale (l'individu. Its autres) et hypothtique (Ǵvénement probable ou assuré) entre l'jndividu to f'objet. Aitsis. plus la distance perçue par f'individu entre luti et l'objut est importante, d'un point de vut geographicpe. comporel, social ou presume. ef plus l'objet sera abstrail et condtura at une moindre 
implication du comportement. Cette theorie a Éf appliquée dans le cadre de l'étude des changements climatiques (Milfont, 2010 ; Spence, Poorlinga, Pidgeon, 2012), les auteurs mettant en évidence que, pour se préoccuper du chongement clinatiqte, if faut réduire celte distance psychologique. Les études condujtes dans co domaine sont réalisées cssentiellement du point de vere de l'individu. Néanmoins, la représentation qu'un individu se formo d'un objet ou d'un événement, bien qu'ítudiée du point de vue individuel, est également féterminée par un contexte social. Ainsi, notre représentation du monde n'est pas individuel le mais sociale, c'està-dire qu'elle fait référence à des modèles collectifs de pensée, des façons d'appréhender le monde collectiventent. Chaque personne, en lien avec ses appartenances sociatos, se construil une représentalion de la réalité qui lut permed de comprendre, d'interpráster, de maîtriser el de rendre signifiant le monde quj l'entoure. La théorie des représentations scciales a pour objet de saisir cette construc tion sociale (Moscovici, 1976). En ce stos, cette approche a trait à la manière dont les idéologies, tes systemes de croyances, de representations, d'évaluations et de normes, autrement die les systemes de régulation, justifient les rapports entre les groupes er les pratiques sociales. Elle vise, par consequent, à comprendre comment se construit la counaissance sociale et comment la punsce seientifique ou, pitus spécifiquenent, des concepts définis scientifiquemenl, sont intégrés dans le sens commun (Moscovici, 1976).

Les représentations socjales correspondent ainsi à différentes formes de connaissances céments informatifs, cognitifs, nomatifs, croyances...) véhiculées par la société et partagétes par un groupe social donné. C'est tue forme de pensée sociale qui permei a l'individu d'appréhender son environmement et quí lui confere une vistion du monde. Les représentations sociales sont donc une construction sociale de la réalite (Jodelet, 1999) qui a pour but de la rendre signifiante (Abric, 2001)

Des études fondées sur la construction sociale du changentent climatigute dans le sens commun montrent un hiveau modérét de connaissances (Cabecinhas, Lázaro, Carvalto, 2008). Certaines études mettent notamment en évidence que les individus ont tendance à associer le changement climatsque au réchauffement (Kcmpton, 1997 ; Lorenzoni, Pidgeon, O'Connor, 2005) et qu'ils se centrent davantage sur les conséquences que sur les causes tu probieme (Cabecinhas et coll., 2008). A ce sujet, les termes a changement " et " rechauffement " sont utilises de raçon équivalente dans Jes médias (Bohn Bertoldo, Bousfield, 20:1). En outre, lorsque le phénomìne est comparé à d'autres problemes socićtaux (comme, par exemple, ta crise Economique), il lend à être considéré comme moins important (Brenchin, 2003 ; Spence, Pidgeon, 2009).

Les représentations sociales sont done fortement contextualiseses et dépendent de l'ancrage social des groupes. Doise et ses collaborateurs (Clémence Doise, Lorenzi-Cioldi, 1994 ; Doise, 1985) défnissent les représcnlations sociales comnte des principes générateurs de prises de position et organjsteurs des differences individuelles, " hies à des insertions sociales spécifigues dans un ensemble do rapports socials et organisant les processus symboliques intervenant dans ces rapports * (Doise, 1985 , p. 246). Les représentations sociales sont définies par leur fouction d'organisation et de lormation des conduites, ainsi que d'orientalion et de rígulation des rapports socialtx. En tant que principes générateurs de prises de position, fes représentations sociales fournissent aux individus des poinls de référence communs qui permettent de reguler les rapports symboliques. Ces prises de position sont gencrees dans les rapports de comnunication. En tant que principes organisateurs, ces systèmes génèrent des ditférences et des variations entre les individus (Clémence et coll., 1994). Autrement dit, sclon l'importance de l'enjeu pour les individus, et selon leur insertion sociale, les prises de position individuelles envers l'objet varieront (Doise, 1992 ; Doise, 1985). Le changement climatique, mis en avant par certains groupes et minoré par d'autres, ctant source d'enjeux et de débats, ne peut etre consideré comme une $\alpha$ réalité objective v et correspond de fạil à une construction sociale de ta téalité propre a chaque groupe. Il existe donc. potentia!tement, autant de réalités que de communaulés concernées par ce phénomène. En conséquence. parce que les préccupations des uns ne sont pas toujours celles des autres, lorsque les individus seronl sollicités ou confrontés à ce sujel, ils acti. veront leurs propres feprésentations dépendantes de leur ancrage social (Doise, 1992); tel est le cas pour des personnes ayant en charge la gestion de t'environnement et, notamment, des agriculteurs, pour qui le climat est un facteur délerminant dans J'exercice de leur mútier.

\section{CHANGEMENT CLIMATIQUE ET AGRICULTURE}

Quand on les inlerroge at sujet du changement climatique, la plupart des agriculteurs prend comme référence des indicateurs proximaux et, particulièrement, lo changement méttorologique, pour essayer de caractériser les risques quj pour- 


\section{MÉTHODE}

\section{Wenquête en France'}

Cette enquête porte sur la période 2009-2011. Elle s'est díroulée dans l'Ouest de la France auprès oe personnes participant à l'é|aboralion de Schémas d'aménagoment et de gestion de l'eau. Le SAGE es un document officiel, quj definit les stratégies d'action et les pratiques à metıre en auve pour préserver localement la qualité ute l'eau. Pour ce faire, des acteurs se regroupent au sein d'une Commisston locale de l'eau (CLE), afin de discuter coltectivement des problématiques tocales. Ces commissions sont constituces de $50 \%$ d'élus (maires, represenlants de syndicals des eaux...), $25 \%$ de représentanls des usagers (associations environnementales, associations de consommateurs, chambre d'agriculture, représentants des industries...) et $25 \%$ de représentants de l'État (preffecture, organismes de gestion de l'eau, Agence régionale de santé, établissements publics, scientifiques...). Des entretiens semi-directits ont été menés aupres de 49 membres de CLE participant à différents SAGE du Grand Ousse (Bas-Léon, Élorn, Odet, Vilane). Parmi les personnes enquêtées : 26 sont des élus, 14 sont des représentants des usagers et 9 sont des roprésenLants de l'Étar, La moyenne d'âge des personnes, majoritairement des hommes (39), est de 55 ans. Parmi les représentants des usagers, 11 personnes représentent des agriculteurs.

\section{E'enquête au Groendand 2}

Une série d'entretiens semi-directifs a été menée auprès de 21 exploitants agricoles, parmi les 46 lermes d'elevage ovin que compte le Grocnland, l'ensemble étant réparti dans la zone Sud du pays ; la moyenne d'âge est de 43 ans. Les participants, essentiellement des hommes (18/21), ont, pour certains, repris l'affaire familiate (13/21), tandis que d'autres, issus de fomilles d'agricuheurs, ont crée leur propre exploitation. Un seul des agriculteurs interrogés n'est pas issu de ce milieu. $L$ 'instalJation ou l'agrandissement des structures agricolts a souvent été rendue possible par l'aide du gouvernement groenlandais, très investi dans l'activitć agricole. La tailte des exploitations est assez hetcrogène : 4 éleveurs utilisent une surface agricole inltrieure à 10 hectures, 5 exploitations sont comprises

1. Les dunntes prégenlécs onl cté recueillies dans le cad re dil programme de recherche CLINASTER, finance par he ptograttrme PSDR (Pour el stur le developpement régiontal) hlp://www 7.rennes.inra.j'/clinwsier/

2. Les clements porlant suf le Groeniand sort issus du peogramme a Green Greenzand $\%$ (ANR CEP\&S 2010). https//sites.googte.com/sileigrecnlaudand cntre 10 et 20 lectares et Jes 12 exploilants restants culivent entre 20 el 40 hectares.

\section{Grilles d'entretien et analyse}

Malgré f'usage d'un guide d'entretien en partie différent, la proximite thértatique des recherches française et grounlandaise a suscilé l'intérêt d'un rapprochement exploratoire des données. Ces enquâtes ont notamment en commun le questionnement sur le changement climalique. Sur ce theme, les personmes interroges mentionnaient, en premier lieu, leurs connaissances et représentations sur le sujer ( $\&$ qu'est-ce que te changement climalijue pour vous? *), puis les causes at conséquences de ce phénomeno sur les ressources naturefles ( « queltes sont lus chuses et conséquences des problèmes identifics precédentmunt? $\%$, plus particulièrement sur hur activité agricole at Groenland (« quel(s) lien(s) avec les pratiques agricoles? $»$ ), et plus spécialement sur fes ressources en eau en France (" quel impuct sur la ressouree en eau ?"). II s'agissait Ĺgalcment de siluer le phénomène dans une échelle de trmps el d'espace (« quelte evolution dans Ie tcmps (passé/présent//utur)? "; " quel impact spatialcment (global/local) ? m). Ces questjons étaient postes à l'enquêté s'il n'y rspondatit pas spontanément.

Les entretiens ont fait l'objet d'une relranscription intégrale el ont ćté soumis à une analyse de contenu mantuelte, thématique et lexicale (repérage du vocabulaire et comptabilisation de la fréquenee de mots). Les thómatiqutes rctenutes pour l'analyse étaicnt les suivantes : connassance/perception du changement climatique (par exemple : réchaulfement, sécheresse, violence des phénomènes, accentuation des phénumènes en nombre, atucune modification...), rôle de la science (confiance/pas confiance, prouve/pas prouve, aggravation scientifigue, désaccord scientifique...), Graiuation spatio-temporelle (ecleelic de temps, local/ global), certitudes/incertitudes (c'est une réalite, je ne sais pas, je re vois pas...) ) liens avec tes ressources naturelles/pratiques agricoles. Pour une meilleture lisibitité et compréhension, nous présenterons d'abord les résultals de l'enquâte menéte en France puis ceux du Groentand ". La discussion mettra en melief les convergences et divergences entrc les deux pays.

3. La presentation des résultats de f"enquêc mense con France est illustroce par des extraits d'entretiens. Cetle restitution a fidele w de la parole de l'interviewé n'a pas été possible pour l'enquête mence au Groentand, dons la mesure où les entretiens ont d́t réalisés en inuit avec l'aida de traducteurs anglais. 


\section{RÉSULTATS}

\section{Enquête dans l'Ouest de la firance}

La représentation des mentes de la Commission locale de l'eau

La définition du changement climatique renvoie essentiellement à la description du phénomène et de ses effets. Il est associé au réchauffement ot à unc évolution des températures. Dans Jes discours, le changement climatique devient Je « réchauffemcnl " $(78 \%)$. Il est Ćgalement défini par des phênomènes de sécheresse (69 \%) ou d'accentuation des phénomênes métcorologiques (orages, tempetcs, ouragans...) (41\%). Une majorité de personnes cite les impacts de ce changement du climat sur la modification de la biodiversité ( $63 \%$ ), la montéc des eaux (59\%) ou encore la fonte des glaces (57\%). Par conséquent, pour la grande majorite des interviewẻs, le changement climatiguc est une réalité, c'est un phénomène qui existe. Ils en sont informés par voie de presse. Le changement climatique, c'est \& ce qu'on lit $*$ ou $\ll$ ce qu'on entend $*$.

En accord avec cetle croyance majoritaire de l'existence du changement climatique (78 \%), les enquêtés tentent de l"objectiver, de le rendre concret. Ainsi, la moitiế des enquêtés (57\%) perçoit des changements du climat localement. Ces modifications s'exprinent notamment à travers la référence aux saisons passées (37\%). Certains enquêtés " voient $\$$ ainsi des ćvolutions du climat dans des saisons plus ou moins marquées, l'absence de neige en hiver, la e taille précoce des châtaigniers ", etc. La question de la saisonnalité ne fait pas consensus. Par consćquent, si lc phénomène esi sans conlcsıe perçu comme global $(76 \%$ ), son ćvaluation loctle apparaît plus complexc et d'autant plus que la croyance majoritaire liêe al « rechauffement " chimatique est peu compatible avec Jes observations du climat dans l'Ouest de fa France : « Je pense que c'est perceptible dans certaines zones déjà arides, le Sahed, Sahara, mais, chez nous, non. Je vois pas de changement. L'eau au mois d'aout l"anne derniere clle était gelée (rires). Donc, on r'a pas senli le réchauffement climatiyuz je ne sais pas si vous avez été à la plage l'année dernière. Dans le temps l'eau était bonne. " (Usager, $n^{\circ}$ 6). De ce fait, pour un tiers des personnes, Je changement climatique g'est pas perceptible localcment.

Cette dimension spatiale est en lien avec la recherche do repères temporcls vćcus, particulièrement à travers la reférence aux saisons passées, par rapporl auxquelles, comme dans la cilation ci-dessus, les personnes comparent l'instant présent. Néanmoins, les changements du climat n' apparais- sent pas perceptibles à l'Echelle humaine (49\%) et sc repercutent sur des siccles, dans l'avenir commo dans le passé, au temps des « mammouths » et des « diplodocus m, au temps où le « Sahara, c'étail du foin * et où lc « Grocnland était vert $\%$. « Je pense que c'est quelque chose d'assez compliqué. C'est ce que je vous disais tout à l'hetrre, c'est vraitment des choses qu'on peut apprécicr dass le temps sur des milknaires en fail, sur des longues périodes $»$ (Ëlu, $n^{\circ}$ 31). Ce raisonnement conduit certains enquêtés à s'interroger sur l'Evolution cyclique naturelle du climat $(47 \%)$, les periodes de refroidissement ou de réchauffement du climal ont 1oujours existé et existeronl toujours.

Ainsi, c'est à travers leurs observations du climal et, plus particulièrement, des phénomènes nútéorologiques, que les enquấs 1entent de rendre visible Je concept de changement climatique, mais l'appréhension globale du phénomène (phénomène planétaire, Evalué sur des échelles de temps élargies) rend la tâche difficile. Alors, une majorité des personnes estime que le changement climatique existe parce que a les seientifiques ic disent $*$. La moitie des personnes affirme ainsi sa confiance en Ia science (57\%) et s'appuje sur efle pour fonder ses croyances. Mais parce que le discours scientifiqte n'est pas toujours perçu clairement, le dotte s'installe, parfois au sein d'un même discour's a Ça rcprésente une menace, ça représente une menace, vague encore si je puis dire, je ne dirais pas incertajne mais je dirais encure pas concrétisée. Je pense que les chercheurs du GIEC notamment, même s'jls sont controverses, il y a suffisamment d'otements aujourd'hui qui montrent qu'on court à la catastrophe " (Élt, $\pi^{\circ} 38$ ). Par consequent, bien que la supromatic de la parole scientifique soit allégú́e, un tiers des persontes met en avant ses failles. Cerlains enquêtés reprochent aux scientifiques de ne pas être d'accord entrc eux el Claude Allegre devient alors la figure de l'opposition, du w scientifique détracteur ». Ces incertitudes accentuent la difficulté à percevoir le phenomène au niveau local et sur tin temps réduit à l'échelle humaine (expression d'hésilations, absence de prises de position : " plus ou moins de venl , " plus ou moins d'orages ", discours énonce sous forme interfogative, emploj du conditionnel...). Le changement climatique est donc bien identifié comme un concept independant des perceptions du climat. Ainsi, si les phénomènes climatiques sont expliqués en référence à des cycles ou des aléas, les causes du changement climatique sont, à l'inverse, clairement identifiées. Ainsi, les rejess de gaz carbonique comme principale cause font peu de doute chez les personnes qui l'expriment (55 \%). Et la production de CO2 est associée 
à l'homme, mêne si sa responsabilité est parfols relativiséc \& (...) Más a la linite, c'est toujours un peu provocateur ce que je vais vous dire, mais qu'est-ce que c'est que le dégagenent de $\mathrm{CO} 2$ des voitures, putrtant je fustigeais son utilisation cout à I'heure, à côté par excmple d'un volcạn qui se met a cracher des volumes impressionnants de gaz ? ${ }^{4}$ (Élu, $n^{\circ} 3$ ).

En outre, te changement climalique est d'autant plus dilficilement perceptible qu'il esı comparé au problème de la ressource en cau : a Alors, c'est difficide d'apprecier le rechauffement climatique parce que c'est une chose qu'on domine certainement pas. Autant sur la cjualité de l'eau on se sent plus actif, plus maître dans la reoherche, dans la mise en place d"objectits te de les réaliser. Dans te réchauffement climatique, c'est quclque chose qui se raisonne au niveau mondial je pense alors on a plus de mal certainement à se sentir capable d'entgager des actions liées spécifiquement au réchauffement climatique. Parce qu'il y a des choses qui nous depassent. (État, $n^{\circ} 24$ ). Le changenent elimatique est, par conséquent, envisagé comtne global et abstrait, à l'inverse des problématiques de l'eau, perçues comme concrètes et locales.

Pour autant, mêtne si ho lich tntre les effets du changement climatique et les problématiques de l'cau traitées, au sein des SAGE, in'cst pas explicile ou direct, l'impact de ce phénomène sur la ressource en eau en gént́ral est perçu. En ce sens, les gestionnaires mentionnent les conséquences d'une modification du climat sur les inondations et lat montée des eaux $(61 \%)$, sur la variabilité de la qualité de l'eau et la perturbation de la vic aqualique (61\%), sur l'assèchement des rivièrus et les étiages sévères (49\%) ou encore sur les zones humides (impact sur ta faute et la flore, par exemple) (41\%).

Le discours des agriculreurs et representants agricoles membres de Conmission locale de l'eat

Enfin, pour les 11 agriculeurs ou fepresentants agricotes interrogés fans cetto enquête, l'association uu rechauffement est ćgalement prépondérante. IIs citent autant les problimes de sécheresse que de précipitation (tempêtes, orages...) et leurs conséquences du point de vue agricole $x$ Ce qui se manifeste plus pour le changement climalique, ici pour l'agriculture jc pense, c'est quand it pleut, $c$ 'est la violence des precipitations certains jours ou des orages forts qu'on n'avail, je pense, pas avant. C'est ça qui accentue le probleme d'brosion dans certains coins du territuire et notamment pour la

4. Referrence a l'Éruption du volcan Eyjafjoli en Islande an 2010 . culture des pommes de lerre s ( $\mathrm{n}^{\circ}$ |4). Mais de même que precédemment, te changemenl climafique semble plus évident au niveau planétaire (Groenland, pôle Nord, Sahel...) que locatement. Les enquêtés pensent majoritairement que les changements de elimat ont toujours existé ; ils ont déja vu ou vécu des années chaudes al des années froides, tel un cycle naturel. De ce fait, les agriculteurs apparaissent sceptiques ou fésignés $* \mathrm{Si}$ on dit que la lempérature de la planète est dépâ... a gagnte quelqutes degrcs, que les entux commencent à remonter, is force de rabatcher, on funit par faire attention je pense. $*\left(\mathrm{n}^{\circ} 9\right)$. En outre, le climat est mis en avant comme une ressolurce importante t: incontournable dans leur gestion quotiditennc, mais il est pris en compte à courl tẹme et peu envisagé à long lerme voire à moyen terme, "Nous, enfin les agriculteurs ont des questions qu"ils si posent vraiment au jour le jour sur la conduite de heur cullure c'est fimite sur le climat, c'est : "demain est-ce que je peux sortir ?" La, ils se poscal les questions, la, en ce moment, ils voient bien guc l'kerbe pousse pas. Bon, ils regardent s"t] pleut pas c'est dujà bien. Donc ça, il fait froid, bon, ils sont en attente des 200 degrés... [rires] depuis le mois depuis, deptis le mois... Enfin on a, on a quefqtes repères climatiques qui permettent de sortir les vaches dans l'herhe. Donc là ils sont sôr, aujourd'hư le climal c'est vraiment une question quotidienne. Mais sur s'adapter à des svolutions à moyen terme, non, non, non, ils font pats. $*\left(n^{\circ} 25\right)$. En outre, les changeanents dans les pratiques agricoles sont mentionnées, mais sins determination possible de la part fiée aux Evolutions structurelles (évolution de la façon de travailler, industrialisation....), de celle lié aux Evolutions du climat. D'aucuns déclaren! mème que dans la région (Bretagne), les années chaudes sont bonnes pour l'agriculture.

En conclusion de cente première partie, la repré sentation du changement climatique apparaît infiueneče par les médias, car peu empreinte de vécu personnel. Les acteurs locaux interrogés ont conscience d'une modification du climat, mais cetle évolution a'est pas ressentis avec certitude. La représentation des agriculteurs ne differe pas fondamentalenent ici de la représentation des autres membres des Commissions locales de l'eau (Ǵlus, repásentants de l'État, reprisentants d'associations d'usagers...). En rúsumé, majoritaitement, les enquêtés, les agriculteurs cornme les autres, estiment que It changement climatique est une réatité. Fls le décrivent à travers le réchauffement el les sécheresses. Mais bien que le changement climatique soit ilfustré au travers du rechauffement on des sécheresses, ce sont davantage les probléma- 
tiques d'inondation, de montese des caux, de perturbation du milieu aquatique qui sont mises en avant, que les problématiques d'éliage ou d'asséchement tion plus empreinte de conoigne d'une représentacrée daus des problé contenus médiatisés qu'anquences du changementiques tocales. Les consédavantage porcugement climatique sont, d'ailleurs, cuéneme perçues at niveau global, même si des Iocentments météorologiques sont parfois decrits Iocalement comme susceptibles d'être en lien avec une modification profonde du climat.

\section{Enquête dans Ie Sud du Grochland}

Par sa siluation géographique, proche des pays industrialises, le Grocnland es! un territoire largement affecte par te réchauffement elimatique or les modslisations climatiques indiquent que le changement amorcé dans cetce région du monde va se poursuivre ati xxie sitele (Masson-Delmotte et coll. 2012). Les Groenlandais sont conscients de ces changements et semblent avoir accepte tes donnés sur le réchauffement global (Buijs, 2010). Plus particulièrement, la population d'éleveurs, travaillant dans le Sud fu pays, est d'altant plus susceptibie, par son activité la confrontant directement aux Eventuels changements environnementaux, de percevoir ces changements. Nous avons done interrogé ces éleveturs groenlandais sur leur perception du clirnat et ses impacts sur leur activité, ainsi que sur leurs stratégies adaptalives face aux changements.

Lorsqu'on teur demande de dire spontanement les mots qui leur viennent à l'esprit pour parler du changement climntique, les agriculteurs groenlandais utilisent des termes qui renvoient à six thematiques, à savoir $1^{\circ}$ le manque de pluie et de neige (22 mentions), $2^{\circ}$ le ressenti du changement climatique ( 18 mentions), $3^{\circ}$ lo réchauftement ( 11 mentions), $4^{\circ}$ la sécheresse ( 9 mentions), $5^{\circ}$ les conséquences nćgatives du changement (7 mentions) et $6^{\circ}$ le changement comme une transformation positive ( 5 mentions).

Comme pour la France (qu'il s'agisse des résultars de la section précédente pour ia Bretagne, ou do ceux de Sérès en 2010), les agriculteurs groenlandais perçoivent massivement to changement el citent ha sécheresse comme référence : 7 f $\%$ notent une baisse des précipitations (pluie el neige). Pour certains (14 \%) le réchauffenent climatique est un phinomènt naturel, et ce changement est reporté à l'échellc globale pour une partie des agriculteurs ( $19 \%$ ).

Lorsque les agriculteurs ont commencé à ressentir le réchauffément climatique, beaucoup (67\%) l'ont interprété comme un élément positif. Ces agricul- leurs $y$ ont en effet perçu l'opportunite de maximiser leurs cultures foumagères tout at long de l'anné, avec la possibilitú de faire deux récoltes au licu d'une seule. Ils ont tegalement perçu l'opportunité de diversilier leur activite, er se toumant vers des cultures tégumières par exemple. Cependant, ce réchauffement a entraîné une sécheresse qu'ils dénoncent $(29 \%)$ comme un facteur provoquant un appauvrissement des sols ct 1'apparition de parasites détruisant les culures ( $48 \%$ ), un probleme avec la quantite d'eau (38 \%) et un appauvrissement de ta quantite de fautie aquatique $(29 \%)$.

$D^{\prime}$ un autre cote, plus d'un tiers des agriculteurs (38\%) notent une évolution dans la fréquence des tempetes, qui sont décrites comme plus récurrentes ct plus violentes que précédemment. Finalement, et contrairement à ce qu'ils avaient espớć, l'ensemble de ces changements entraîne des conséquences economiques dommageables pour les éleveurs groenlandais qui, ne pouvant plus produire altant à cause des épisodes récurrents de secheresse, se trouvent dans l'obligation d'acheter du fourrage en provenance du Danentark (24\%). Des conséquences sociales sont également dócrites : la díminution des pertiodes de gel cntraîne l'impossibilité de se déplacer sur les ljords, compromettant ainsj les relations sociales ontre les habitants des fermos isoletes, dont l'acces se faisait auparavant en motoneige pendant thiver (24 \%).

Par ailleurs, les Groenlandais semblent avoir du mal à se projeter dans le futur et, par conséquent, à se représenter l'évolution du climat et de leur activite professionnelte dans les années à venir. Daris leur discours, ils rappellont que les Inuits ont longlemps été un peuple nomade, qui a toljours su faire preuve de capacités d'adaptation face aux changements rencontrés à travers les âges Il n'y a atcune raison qu' $i$ ] en soit autrement dans l' avenir : les agriculteurs, en reférence à ce passé, estiment pouvoir s"adiapler de la mame manière au changement climatique, en adoplant des stralégies d'adaptation ponctuelles, comme l'utilisation de systèmes d'irrígation drainant l'eau des lacs glaciaites (43 \%) ou encore diversifier les espèces prairiales $(81 \%)$. La moitié des personnes interrogées, particulierement celles ayant des grandes exploitations, utilise d'otes et duja ce type d'irrigation. Enfin, des stratégies plus structurelles, comme le test de differentes cultures est envisagé $(33 \%)$ voire pratiqué à l'échelle de l'explotiation, mais cette adaptation ne semble pas encore se développer à grande échelle. Ainst, les discours portant sur la representation de t'activité dans le futur sont à fa fois variés et évasifs : certains $(24 \%)$ indiquent ne pas pouvoir faire de prévisions, ce qui ne leur pose pas de problème particulier. 
Pour d'autres (24\%), la situation elimalique paraî inguiélante, mais finalement une grande partic do l'échantilon (43\%) rapporte spontasement une capacitó culturelle d'adnptation face à ce changeFent et se montre plutôt optiniste face à l'avenir. Enfin, the partic des bleveurs ( $38 \%$ ) soubaite que
Je réchauflement et la sécheresse ne s'aggravent pas dans le Tuktr. Les résulıals relièrent globalement une difficulté de projection dans l'averitr, peu d'anticipation et, lorsqu'elle est présente, uniquement à court terme, c'est-à-ctíe dans le dúlai fraximum d'une année à venir.

\begin{tabular}{|c|c|c|c|}
\hline & \multicolumn{2}{|c|}{ En Franco } & \multirow{2}{*}{$\begin{array}{l}\text { Au Groanland } \\
\text { Agricutteurs }\end{array}$} \\
\hline & Gastionnaires de l'eat & Agriculteurs & \\
\hline $\begin{array}{l}\text { Description du } \\
\text { phéftomène ot } \\
\text { de ses effets }\end{array}$ & $\begin{array}{l}\text { Réchauffement, sócheresses, } \\
\text { accentuation des phénomènes } \\
\text { météorologiques. } \\
\text { Modificalions de la biodiversité, } \\
\text { montée des eaux, fontes des } \\
\text { glaces. }\end{array}$ & $\begin{array}{l}\text { Sécheresses et accentua- } \\
\text { tion tes phénomènes crageux } \\
\text { (précipitations...) }\end{array}$ & $\begin{array}{l}\text { Manque de pluie. Réchauffe- } \\
\text { ment, sécteresses, fréquence } \\
\text { des tempêtes. Changement } \\
\text { dans fa taune, conséquences } \\
\text { économiques (achat de fourrage } \\
\text { et moutons plus petits). } \\
\text { Conséquences sociales (rela- } \\
\text { tions isolées entre habitants). }\end{array}$ \\
\hline $\begin{array}{l}\text { Localisation } \\
\text { deg effets du } \\
\text { changement } \\
\text { climatique }\end{array}$ & $\begin{array}{l}\text { Phénomène global, planétaire. } \\
\text { Enquêtés partagés entre: } \\
1^{\circ} \text { ceux qui percoivent le } \\
\text { phénomène localement à travers } \\
\text { la référence aux saisons passées } \\
\text { fabsence de neige en hiver, taille } \\
\text { précoce des plants...) } \\
2^{\circ} \text { ceux qui ne pergoivent pas de } \\
\text { modifications du climat. }\end{array}$ & $\begin{array}{l}\text { Flus évidents dens d'autres } \\
\text { endroits de la planète (Groen- } \\
\text { land. pöle Nord, Sanel...) que } \\
\text { lacalement. }\end{array}$ & $\begin{array}{l}\text { Phénomène reporté a l'échelle } \\
\text { globale. }\end{array}$ \\
\hline $\begin{array}{l}\text { Le temps de } \\
\text { P'évolution du } \\
\text { cllmat }\end{array}$ & $\begin{array}{l}\text { Temps long, les changements se } \\
\text { répercutent sur des siècles. } \\
\text { Evolution naturelle di cyclique? }\end{array}$ & $\begin{array}{l}\text { Les changements de climat ont } \\
\text { toujours existé. } \\
\text { Cycle nqturel. }\end{array}$ & Phछ́nomène naturel. \\
\hline $\begin{array}{l}\text { Référence à la } \\
\text { science }\end{array}$ & $\begin{array}{l}\text { Conforte l'existence du climat. } \\
\text { Une majorité fait confiance. } \\
\text { Un tier's met en doute (experts } \\
\text { en dísaccord, aggrava- } \\
\text { tion du phénomène par les } \\
\text { scientifiques...) }\end{array}$ & & \\
\hline Les cauges & $\begin{array}{l}\text { Le rejef de gaz carbonique (et } \\
\text { donc « indirectement * l'homme) }\end{array}$ & & Cycle naturel. \\
\hline
\end{tabular}

Le changement climatique et les ressoufces entronnementales

Ia ressource en eat

Les tiens

Les impaets perceptibles

\begin{tabular}{|c|c|c|}
\hline $\begin{array}{l}\text { Inondation, montée des eaux, } \\
\text { eflages séuétes, modification } \\
\text { de la qualité de l'eau et de la vie } \\
\text { aquatique. }\end{array}$ & $\begin{array}{l}\text { Le climat est une ressource } \\
\text { importante. } \\
\text { Prise en compte à court terme } \\
\text { (mótéo) }\end{array}$ & $\begin{array}{l}\text { Peu de projections dans la futur. } \\
\text { Stratégies d'adaptation } \\
\text { envisagées. }\end{array}$ \\
\hline $\begin{array}{l}\text { Difficiles à évaluer, échelles de } \\
\text { temps et d'espace différentes. }\end{array}$ & $\begin{array}{l}\text { Erosion des sols par la vióence } \\
\text { des précipitations. } \\
\text { Le réchauffement, un élément } \\
\text { parfois positif. }\end{array}$ & $\begin{array}{l}\text { Parasites dans les culłures. } \\
\text { Appauvrissement de la faure } \\
\text { aquatique. } \\
\text { Réchauffement perçu au début } \\
\text { comme un éiément positif. }\end{array}$ \\
\hline
\end{tabular}

Tahleau 1. Synthèse de la représentation sociale du changement etimatique. 


\section{DISCUSSION ET CONCLUSION GÉNÉRALE}

L'ensemble des résultats présentés ci-dessus fait apparaître une population majoritairement sensible au changement elimatique, qui met pet en doute son existence, malgré de relatives incertiludes quant a la projection de ses consépuences at long terunc. Pour la majorilé des enquêtes, quel que soil te contexte. le changement climatigue ust 1'tel ut s'actualise at fravers d un rechtuffentent des températures. Ces résultats sont concordants avec ta plupart des études menées sur ce thème (Bohn Berfoldo, Bousfield, 20I I ; Cabecinhas et coll. 2008 ; Kempton, 1997 ; Lorenzoni el coll., 2005). En matière de conséquences, ke changement climalique est associte à des periodes de sécheresses, un bouleversement des phénomènes métécorologique en nombre ou en intensite. .

De ce fait, tentant de s'approprier ce concept, de l'objectiver (Moscovici, 1976 ; Doise, 1985) ot de te rendre concret, les enquêtcs lendent à rechercher des efTets d $d^{\dagger}$ une modification profonde du climat dans leurs observations locales des phénomènes mctcorologiques (plus dc périodes de sccheresses, intonsification des tempêtes...). C'est ici que des distinctions apparaissent entre nos deux populations seion leur ancrage geoclimatique (Doise, 1992). Dans l'Ouest de la France, le réchauffement des temperatures el les phénomènes de sécheresses ne sont pas directement observabics. Or, c'est ce qui caractérise le plus le concept de « changertent climatique ". Les enquêtés ne doutent pas de sort existence à un rivea planétaire, mais fe phénomène apparaît localement peu concret. Un tjers des enquêtés français exprime explicilement ne pas voir de chamgenent climatique. A l'imverse, les problèmes pouvant être en lien avec ce phénomène (sécheresse. pénurie d'eau...) sont clairument identifics localement che $z$ les éleveurs groenlandais. Aiusi, a l'exemple des résultats des Iravaux de Lémery et colj, (2005), selon les conditions géoclimatiques, la perception des nodifications du climat varte. En conséquence, selon les régions, le changement climatique peut etre defini soil comme un risque, caractérisé par son impact planétaite et son invisibilite, soit comme une cvolution environnementale a laquelle il faut faire face. En d'autres termes, si Jes Français considèrent que le changement ne se ressent pas à l'échelle locale, les Groenlandajs, quant à cux, perçojven! ce changement localement, mais ne le considèrent pas pour autant comme upe mise en danger de leur activité.

Pour les gestionnaires français, scule, la science pourrait maf̂triser le risque associé at changement climatique, mais elle apparaît ellc-même impuissantc ot contradictoirc face à ses propres incertitudes soulignées (Beck, 2008). De ce fail, placer le changement climatique comme étant " éloigné de soi », dans le temps et dans l'espace, permet de se rassurer et de se protéger de sentiments inconfortables qui necessiteraient une modification des comportements. Cet éloignement du problème est particulierement saillant en rapport aux problematiques locales de l'eau. Ainsi, même sỉ les personnes interrogées croient majoritairement en la tćalité d'une modification du climat, onême si elles retienneat des liens avec la ressource an eau en générai, la comparaison des conséquences globales el à long terme de ce phénomènc, aux conséquences immédiates et saillantes das problèmes locaux likes à l'ear, fait que ces derniers sont pergus comrne plus sérieux et pltus importants (Michel-Guillou, 2012). En co sers, les changements climaliques ne constituent pas une priorité et ne sont done pas envisagés comme un risque imminert. Cette comparaison entre la problématique de l'eau et celle du changement climatique, il]ustre hicn le positionnement en reffércnce à la distance psychologique (Liberman, Trope, 2008 ; Trope, Libctman, 2003). Lc probtène de la qualité de l'eal, notamment en Bretagne" sest perçu comme spatialement et socialement proche, autrement đit, ce problème est circonscrit dans l'espace régional el touche toute la population bretonne (Michel-Guitlou, 2011). C'esi un problème reconnu et avért́ el c'est aussi un problène siltué tempore]lement ; l'émergence du probleme est dateco et sa résolution dans l'avenir est envisagce. Même st le temps de recouvrement est long, le processus d'amblioration de la qualité de l"eau $a$, selon certains gestionnaires, dejầ commencé. À l'inverse, sclon les enquêtés trançais, te changement climatique touche des populations éloignées socialcment er géographiquement. C'est un phénomène par rapport auqued les conquêtes estiment ne pas avoir de prise temporellement. Lc changenent climatique est, par conséquent, plus conçu comme une réalité scientifique, relayce par la communatú des chercheurs, qu'une réalité expếrientielle vécue par tout un chacun sur le terriłoire. Ses conséquences sont encore perçues comme incertaines. À l'jnverse, au Groentand, là oì le rechauffement es1 ressenti, Ies éleveurs discernent bien le changement climatique et ses conséquences sur le territoirc, mais ils placent egalement une distance psychologique qui ne se traduit pas ici en fonction de ta distance spatiale. Elle se lìt à travers la distance temporelle, qui rend visible l'êvolution du rechauffernent (inscription

5. Voir le site Gest Eat: : http:/www.gesleau.eaufance. 
dans le passó), mais sans référence à une cchelie temporelle plus large, sans projection dans l'avenir. Cette attilude s'explique strrout par d'un fort sentiment d'efficacilć perçue et, donc, de contrôle des élémerts naturcls. La reconnaissance d'une proximite des problemes accentue le sentiment de controle perçu sur ces problemes (Garcia-Mira et colt. 2005 ; Moser, 2009). Et c'est exactement ce sentiment qui est exprime par les gestionnaires de l'eau français sur la ressource dont ils ont la gestion. Ainsi, en experimentant directement les conskquences du changement sur leur territoire, les agriculteurs groenlandais semblent plus enclins que les Français et, notamment, les agriculteurs, à definir des strategies d'adaptation.

Comme nous venons de le souligher, sur l'aspect temporel et, essentieliement, l'borizon futur, les Français et tes Groenlandais se retrouvent. Les enquetés expriment leur difficutte à situer le problème dans un espacc-temps. L'ensemble des agriculteurs, Français et Groenlandais, fon cetat d'une difficulté de projection dans l'avenir, liéc à la conception gu'ils ont de leur propre capacité à faire face. Pour les agriculteurs groenlandais, par exemple, en voyant l'irrigation comme solution adaptative, ils ne se posent pas la question de la pérennité de ce type de pralique. Or, des expériences similaires au Canada en ont montré les limites dans une échelle temporelle très courte (Furgal, Martin, Gosselin, 2002 ; Ford, Smit, Wandel, MacDonald, 2006) : après trente ans d'utilisation, la rarćfaction de la pluyiométrie ne perfnet plus d'cmployer l'eau pour les activités agricoles. En outre, l'observation des changenents envirofnementaux lićs au changenent climatique sork les memes d'un tertitoire ì un autre lorsque celuj-ci est silut dans une zont fortement exposce. Aissi. les communautés himalayennes (Chaudhary et coll., 2011), tjbélaines (Byg, Salick, 2009) ou cneore cantidiennes (Berkes, Jolly, 200t, Furgal el côl., 2002) décrivent le meme type de changement dans leur environnement. La perception de ces changements repose essentiellement sur une modification de l'écosysteme (faune, flore), sur un dériglement climatique (saisons decalées, moins de pluie, de neige...) el sur une impossibilité de prévoir l'śvolution du changentent. Les stratégies developpses pour faire face à ces differents changements sont également similatres et tendent davantage vers des adaptations ì court terme (par exemple, irrigation pour les uns, décatage dans la saison de chasse et de pêche pour les autres). Bien que situtes dans des contextes gtographiques différenciés, ces différentes cultures ont un point commun : \{eur capacité d'adaptation dans un epvironnement incertain (Berkes, Jolly, 200 l).

Dans ce cadre, whe hypothèse sur Je type d'adaptation émerge. Les Groentandais perçoivent la transition climatique qui s'operre, mais se raccrocheraich à leur histoirc culturelle pour meltie à distance ce phénomène, alors que les agriculteurs français, qui ne perçotvent pas le réchauffement (moins marque gu' au Groenland) mettraient celte transition climatique a distance. Ces attitudes entraineraient, pour les deux populations, une incapacitć de projection temporelle suffisamment ćloignée. Par constíquent, its adopteraient, matgré les variations géoclinatiques, des stratégies d'adapuatjon similaites (ponctuelles al non structurelles). Ces deux types de stratégies renvoicnt quelque peu aux stratégies de traitements de l'information (Chaiken, Liberman, Eagly, 1989). Ainsi, pourrait-on faire un parallèle entre tratement heuristique ef adaptation ponctuelic, el traitement systematique et adaptation structurelle. La notion d'incertitude renverrait, dans ce cas, les agriculteurs à se référer à des informalions partielles pour élaborer leur's stratégies d'adaptation, tandis qu'ił conviendrait de se référur à une analyse holistique de la situation pour aboutir à une strategie d'adaptation structurellc, davantage tournéc vers l'avenir de l'activité. Mais commeni arriver it ce niveatu de traitement, lorsque les dontees, scientifiques et expérientieltes, sont interprétées différemment et purlois même de manière contradictoire? Une analyse de la croyance en l'information scientifique ou expérientielle, en lien avec les strategies d'adaptation, serai à approfondir dans de futures recherches. L'hypothèse serait, ators, d*analyser dans quelle portion et de quelle manière la confiance dans sa propre perception ou la confiance en la science favoriserait on, au contraire, freinerait la projection dans l'avenir et, conséquemment, la gestion des ressources environfementales et l'atnúnagement des activités qui y sont lices. 


\section{RÉFÉRENCES}

ABRIC (Jon-Clatude)- Les représenlations suciales: aspects theoriques. dans Abric (J-C.), Protiques rocidales et représentation, Paris, Presses universìtaires de Fronce, $2001+$ p. $11-35$

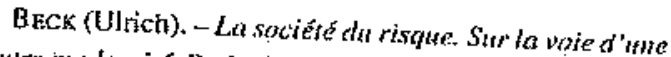
autre molemisé, Paris, Fiammarion, 2008.

Bekkrs (Fikret), Jolił (Dyanna)-- Adapting to climate change: Social-ecological resificnce in a Canadian Westem Arctic Community, Conrermation Forleggy $5,2,200$ I , p. 18.

BoIin Bertol.do (Raquel), Bousfict.d (Andréa Barbara S.)- Representitions socjales du changement climatique : effets de contexic el d'implication. Temas em Psicologia, 19, 1, 2011, p. 121-i37.

BRENCHiN (Sleven R)-Comparative public opinion and knowledge on grobal climalic change and the Kyoto Protocol: The U.S. wersus the world?, Intemailonal .atrnat of Sociology and Soctal Policy 23, 2003, p. 106-34.

Buss (Cumera).- Inuit perceptions of climate change in East Greenland, Inuir Shoties, 34, 1, 2010, p. 39-54.

Bro (Anja), SALick (Jan)-Local perspectives on a global phenomenon. Clinate change in Easiem Tibetin villatges, Global Envimmental Change 19, 2, 2005, p. 156-166.

Cabecinhas (Rega), Lazako (Alexandtr), Calvatho (Anabela)- thedia uses and social representitions of climate change, dans Carvatho $(A$,$) , Communicating$ Climate Chahge: Dircoursas, Meditations ant Percepthons, Braga, Centro de Estudos de Comunicação c Socicdade. Universidacie do Minho. 2008, p. 170-189.

Chajk:in (Shclly), Llabrann (Akiva). Eagly (Alice H.)- Heuristie and systemitic processing within and beyong the persuasion context, dans Utchan (J. S.), Bargh (J. A.), Uninended Thought, New York, Guitlord Press, 1989, p. 212-252.

Chaudhary (Pashupati), Ral (Sumato), Wamgor (Sidathant), Mao (Akai), Regman (Nishat), Chet rRi (Sagtosh), Bawn (Kamaljit S.)-Consistcncy of locat perceptions of climate change il the Kangehenjenga Hinalaya lands sape, Cinren Science, 101, 4, 201 1, p. 504-513.

Clámante (Main), Dolse (Willem), Lokenzl-CioloJ (Fabio). - Prises de posilion el principes organisateurs des rupresentations sociales, dans Guimelli (C.), Silweturer ef Mansformation.r der reprérentutions socioler, Neuchălel, Delacliaux et Niestle, 1994, p. 1 j 9-152.

Dorse (Willen).- Les représcntalions sociales : définj- tion d'un concept, Commexions, 45, 1985, p. 243-253.

DoIse (Wiljem).- L'tucragt detns les éludes sur les representations socialos, Wulletin de prychologic, 45, 405 , 1992, p. 189-195.

FoRd (James D.), SMIT (Barry), WANDEl (Johanna), MaCDONALD (John)- Vulnerabilicy to cfimate change in Igloolik, Nunavut: What we can tearn from the past and present, Folar Recond, 42, 221, 2006, p. 127-138.

Furgat (Cliristopher), Martin (Danieb), Gossel (Pierre),- Climate Clange and Health in Nunavik and Labrador: Lessons from Inuil Knowledge, dans Kruprik (I.), Jolly (D.), The Earh is Faster Nont hatigenous Obsempins of Awtic Entronmemal Change. Washington, D.C., Arctic Research Consortium of the Unted Stales, Mrctic Siudies Centre. Snithsonian Institution, 2002; p. 266-300.

Garcla-Miha (Ricardo), Real (J. Eulogio), ROMAY (Jose) - Temporal and spatial dimensions in the perception of environmental problems: An investigation of the concepl of environmental byperopia, Interiotional Jowrial of Psychology! 40, 2005, p. 5-10.

GJEC (Grotpemen! intcrgouvernemental sur l'Evolution du clima1)-Bilan 2007 des changenenrs climar-

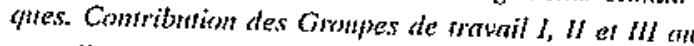
quatrième Rapport d'éwalitation dh Groupe d"experts intergortementenat wa l'éwhtion dit climat, Genève, 2007.

[PCC (ntergovernmental Panei on Climate Change).Climale Change 20/3. The Physical Science Bomis, New York, Cambridge University Press, 2013.

JODELET (Denist).- Representations sociales : un domaine en expansion, dans Jodelel (D), Les représedrtafion, sotialew, Paris, Presses universilaires de France, 1999, 6" Ed. p. 47-78.

KEMPT ON (Willet).- How the putic views elimate change, Enwiromment, 39, 1997 , p. 12-21.

Legendre (Alain).- Psychologie environnementale: de l'étude des systèmes complexes personne-environne. ment à fa préservation el l'améliontion du cadre de vic. Prychoiggie et socítété 8, 2005, p. 7.24

Lemery (Bruno). Ingrand (Stáphime), Detjey (Benoî), Dégrange (Bealnice)-- Agir en situation d'incerlitude : le cas des elcveurs de bowins atlatiant, Eomomie rimale, 288, 2005, p. 57-69.

Lrabrman (Nira). Trope (Yacov).- The psychoJogy of transcending here and now, Science, 322.2008, p. $120 \mathrm{~J}-\mathrm{i} 20 \mathrm{5}$. 
LI VERMAN (Dinna)- From uncerain to uncquivocal. The IPCC Working Graup I Repolt: Clinate Change 2007. The pilysical science besis, Envinmmen, 49, 2007, p. 2B-32.

Lorenzonl (lfün), Pingeon (Nick F.), O'Connor (Robert E.)-Dingerous climate change: The roic tor risk useurch, Risk Analy.sis, 25, 6. 2005. p. 1.187-1398.

Massow-Drgmotte (Vhlérie), Swingloouw (Djelier), LANDAIs (Amatlle), SEldLinkizantz (Marit-Solveig), Gauthiter (Émitie). Bichet (Vincent) el colt. GreenIand climate change: Fram the past to the future. Wifey Interdistiplinary Reviews: Climate Change, 3, 5. 2012, p. 427-449.

Michíc-Gulleou (Élisabeth)- L.a construction sociale de la ressource en enu, Prariques prychulogighes, $17,2011,0.219-236$.

Michal-Gullou (Élisabeth).- Changament climetiqute es ressotrce en eau. Quelte reprissentation du changement climatique ? Quedle prist to comple dans la gestion jocale des problumes liés à la ressource en eat ? duns Merot (P), Dubreuil (V), Delahaye (D), Desnos (13). Chatgement rimatigue dans l'OHest Evathatim. imprets, perteprions, Rennes, Psesses univursiaires de Rennes, 2012, p. 387-398.

Milfont (Thciano L.)- Global warming, climale charge and human psychology dans Cortal-Verdugo (V), Gareia-Cadena (C. H.) Frlas-Arment (M.), PsychoIogial appluaches o sustainabilisy Current reuds in theory, researh and practice, New York, Nova Science Publishers, 2010 , p. 19-42.

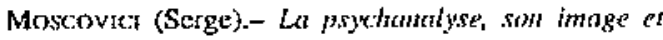
sim $/$ mblfi, Paris, Presses universítaires de France, 1976.

Mosede (Oabriel).- Fy phologie envirmmentenrale. Les relations homme-anvinhntmen. Bruxclles, De Boeck, 2009.
Oneskes (Nammi)- The Scientilic Congensus on Climate Change, Srictee, 306, 2004, p. 1686.

SÊtès (Ctaire)- L'agriculture face au changentent climatique en zonte de montagnc : Guolutions clinatiejtes, perceplion des cleveurs et strategies d'adaptation des systèmes fourtagers, fourrages, 204, 2010, p. 297-306.

Spence (Alexi), Pidgean (Nick).- Psychology, climale change and sustainable beluvior, Envimommen, $51,2009, \mathrm{p}, 9+1 \mathrm{~g}$.

Srence: (Alexa), Poortunga (Wouter'), PtDiedn (Nick).- The psychological distance ol climale ctange, Risk Analyis, 32, 20:2, p. 957-972.

STOKOts (Daniel), ALTMAN (Irvin),- Intiudustion, dans Siokols (D.), Altman (1.), Homdbouk of eminmmenal prychology, New York. Witey \& Sons, I987, p. I-4.

Trope (Yacov), LibekmaN (Nira)- Temporal construal, Prychological Revieus, 1to, 2003, p. 403-42 I .

TROPE (YhCOV), LIHERMAN (NiYa), WAKSLAK (Cheryl J.).- Construal levels and psychotogical distance; Erfects on representation. pediction; cvaluntion, and behavior. Jourial of Cousuner Psychology, 17, 2007, p. 83-95

Uraelt. (David)- "The psycho-spatial dimension ol global environmental problems, Jonnat of Eimmomental Sxychologw, 20, 2000, p. 307-318.

Weaer (Elke), Sorka (Steve)-Production and pricing decisions th casly-crop tarming eftects of decision trails and ctimate change expecintions, dass jacobstn (B.). Pedersen (D.), Christensen (1.), Rasmussen (S.). Fommers' decinion making: A dexciptive apprard, Copenhagen, European Association of Agricultural Economists, 1994. p. $203-2 \mathrm{~EB}$. 